\title{
LA EVALUACIÓN COMO ESTRATEGIA DE CAMBIO DE LA EDUCACIÓN SUPERIOR*
}

Luis YARZÁBAL**

\section{Resumo}

Este artigo assume o ponto de vista da UNESCO a respeito da importância da educação de qualidade para enfrentar os desafios do mundo moderno e formar cidadãos capazes de construir uma sociedade mais justa, solidária, respeitosa dos direitos humanos e da eqüidade no uso do conhecimento e da informação. A avaliação institucional pode converter-se na melhor ferramenta para guiar a transformação da educação superior. O IESALC (Instituto Internacional para a Educação Superior na América Latina e Caribe) está priorizando a discussão de parâmetros de avaliação que tomem em conta as características da educação superior da Região. Para o autor, dentre outros parâmetros, a avaliação deve ser necessariamente participativa e global e tem como etapas imprescindíveis a sensibilização, a auto-avaliação, a avaliação externa por pares, a tomada de decisões e a re-avaliação permanente. Finalmente, o autor anuncia os possíveis apoios do IESALC-UNESCO a iniciativas que promovam a avaliação institucional na Região.

Palavras-chave: Educação Superior, IESALC-UNESCO, qualidade, avaliação institucional, parâmetros de avaliação institucional.

Los estudios solicitados por la UNESCO y las numerosas consultas convocadas por la organización en el transcurso de la presente década han acumulado información que apoya la hipótesis que sostiene que la calidad de vida de las comunidades humanas dependerá cada vez más de sus niveles cultural, educativo y científico, los cuales se relacionan estrechamente con la calidad y pertinencia de sus instituciones y sistemas de educación superior (Delors et al., 1996; CRESALC, 1996 y 1997; UNESCO, 1998)

Al mismo tiempo, diversos indicios revelan que el proceso de globalización, en su impacto sobre la educación superior, está conduciendo a un modelo mundial estandarizado y probablemente hegemónico, en el cual el Estado se retirará progresivamente del escenario, dejándolo libre para que operen en él las fuerzas 'reguladoras'del mercado.

Como lo señalan Jacques Attali et al. (1998), "En ese modelo (donde la educación superior dejaría de ser una carga para la sociedad para volverse

- Trabajo presentado en: "Seminario sobre Evaluación", Facultad de Educación, UNICAMP, Campinas, Brasil (16 - 18 de noviembre de 1998).

- Pesquisador do Instituto Latino Americano para o Desenvolvimento - ILAEDES. 
una fuente de ganancias para las empresas), la emulación y la competencia se implantarían a todos los niveles".

De generalizarse esta concepción, tendríamos como resultado una organización piramidal de la educación superior que ofrecería en su cúspide una enseñanza de excelencia, destinada a los estudiantes dotados de elevado poder de compra, y en su ancha base, una educación de masas, cada vez más deteriorada. En la primera se concentrarían los capitales, las instituciones de excelencia, los profesores más calificados y los estudiantes mejor preparados por los niveles anteriores de los sistemas educativos. En la segunda, imperarían las restricciones financieras, pulularían las instituciones de baja calidad y se estacionarían los estudiantes de los sectores pobres de la sociedad.

En América Latina y el Caribe ya se está dibujando un paisaje institucional que reproduce esta imagen, mostrando en varios países sistemas de educación superior compuestos por instituciones que configuran dos modelos asimétricos. Unas pocas, privilegiadas, han logrado las condiciones necesarias para ofrecer una educación de excelente calidad, a condición de ser rigurosamente selectivas y de disponer de fuentes seguras de financiamiento. Otras muchas, entre las cuales se incluyen cada día más universidades públicas, están siendo obligadas a proporcionar una enseñanza de "supervivencia", encargándoseles la atención del explosivo crecimiento de la matrícula estudiantil, mientras se les cercenan sus posibilidades de desarrollo mediante el mecanismo de la reducción sostenida e implacable de los recursos financieros.

Los diferentes actores sociales que respondieron a las convocatorias efectuadas por el CRESALC en la región han proclamado, primero, que "la educación en general, y la superior en particular, son instrumentos esenciales para enfrentar exitosamente los desafíos del mundo moderno y para formar ciudadanos capaces de construir una sociedad más justa y abierta, basada en la solidaridad, el respeto de los derechos humanos y el uso compartido del conocimiento y la información"; y, segundo, que "la educación superior constituye, al mismo tiempo, un elemento insustituíble para el desarrollo social, la producción, el crecimiento económico, el fortalecimiento de la identidad cultural, el mantenimiento de la cohesión social, la lucha contra la pobreza y la promoción de la cultura de paz" (CRESALC, 1996).

Pero, paralelamente, los mismos actores han señalado que "la educación superior (sólo) podrá cumplir tan importante misión en la medida en que se exija a sí misma la máxima calidad, para lo cual la evaluación continua y permanente es un valioso instrumento" (CRESALC, 1996).

Por estas razones hemos atendido con especial interés la invitación a participar en este seminario y a contribuir a iniciar desde esta casa de estudios un programa destinado a formar recursos humanos de alto nivel para crear y sostener una cultura de evaluación transfromadora en las instituciones de 
educación superior de la región. La UNICAMP ha sido pionera en la puesta en práctica de la evaluación institucional en el Brasil, aportando, con el trabajo colectivo de sus integrantes, instrumentos metodológicos, procedimientos y conceptos, que actualmente son de extraordinario valor para apuntalar el proceso de cambio y desarrollo de la educación superior de la región (DIAS SOBRINHO, 1994).

Todos los que estamos acá conocemos de primera mano la difícil coyuntura en que se encuentran las universidades públicas latinoamericanas y caribeñas. Estas entidades, forjadoras del sentido de nación en cada uno de los países de la región, generadoras de los cuadros profesionales, políticos y empresariales que han viabilizado el desarrollo de nuestras naciones, defensoras valientes de los derechos humanos, la democracia plena y la integración social, se encuentran hoy seriamente amenazadas y requieren nuestra colaboración más decidida.

Las universidades reciben presiones de actores muy variados, destacándose entre ellos: los estudiantes, que requieren acceso, programas y servicios adecuados a sus necesidades e intereses; los sectores productivos privados, que demandan egresados con las calificaciones que exige el cambiante mundo del trabajo; los gobiernos, que insisten en hacer más eficiente el uso de los menguantes recursos financieros que les asignan; los organismos financieros internacionales, que alegan que la inversión en educación superior no es lo suficientemente productiva como para hacerla prioritaria; y también la sociedad en su conjunto que, tal vez ingenuamente, espera de la universidad respuestas ágiles y creativas a los cruciales problemas de desarrollo económico y social que deben ser resueltos por múltiples actores y no sólo por las instituciones de educación superior.

Sin embargo, las universidades no pueden permanecer impávidas frente a esas presiones y a los desafíos que se le están planteando. Por el contrario, deben asumir una posición proactiva y propositiva, abandonando la postura reactiva y defensiva que las ha caracterizado en las últimas décadas, particularmente frente a las propuestas que hacen en la actualidad los organismos financieros internacionales.

De acuerdo con lo preconizado por la UNESCO, tendrán que prepararse para responder a los requerimientos de la formación permanente, a los cambios generados por la revolución científico-tecnológica y a la necesidad de convertirse en instituciones altamente eficientes en el manejo de recursos públicos.

En tal contexto, la evaluación puede convertirse en la mejor herramienta para guiar la transformación que debe realizarse al interior de cada institución de educación superior. Puede ser la ocasión propicia para redefinir la misión particular de cada una de ellas, para establecer objetivos y metas y elaborar instrumentos que permitan la verificación del cumplimiento 
de éstas. De ese modo, puede constituir un excelente ejercicio formativo a todos los niveles y una manera eficiente de encarar la relegitimación frente a la sociedad. Debe, en suma, ser encarada y adoptada como la principal estrategia para el cambio.

Dicho lo que precede, es oportuno reconocer que hay muchas formas de acercarse a un proceso evaluativo. Generalmente la mera mención de la palabra genera suspicacia e incomodidad, sobre todo cuando muchas instituciones nunca han establecido mecanismos claros, participativos y consensuados para examinarse a sí mismas. Es natural que frente a las exigencias que hacen hoy tanto gobiernos como organismos financieros - ambos cuestionados con creciente frecuencia y cobertura por sus prácticas injustas y éticamente cuestionables - los universitarios respondan con vehemente renuencia a la idea de ser evaluados. Los criterios, la forma, la identidad del evaluador, son escrutados a fondo y el ejercicio en sí mismo es resistido hasta donde sea posible.

En el momento actual disponemos de una gran variedad de modelos de evaluación. La mayoría se construyeron en los países desarrollados. Algunos de ellos han sido aplicados en países de nuestra región. Otros, particularmente en los últimos años, han sido generados en ella, pero aun no se les ha sometido a evaluación. La gran variabilidad institucional y programática hace desaconsejable el intentar elaborar un modelo único para la región.

Por ello, la UNESCO, a través de su recientemente creado Instituto Internacional para la Educación Superior en América latina y el Caribe (IESALC), ha decidido dar prioridad a este asunto y está convocando a especialistas provenientes de aquellos países de la región que han desarrollado procesos de evaluación y acreditación de sus sistemas, instituciones o programas de educación superior a intercambiar informaciones y experiencias con el fin de armar juntos modelos alternativos de evaluación. Esta reunión, en la cual participarán expertos de Argentina, Brasil, Chile, Colombia, México, Puerto Rico y Venezuela, tendrá lugar en San Juan de Puerto Rico, del 12 al 14 de enero del próximo año, y pondrá a prueba nuestra hipótesis de que se pueden elaborar modelos que respeten las especificidades de los programas, las instituciones y los sistemas de la región y sus subregiones, así como sus relaciones con el entorno social de cada uno de ellos.

Nuestra experiencia nos induce a pensar que la evaluación puede ser transformadora en sí misma, constituyendo una extraordinaria oportunidad de renovación institucional y profesional para sus cuerpos docentes, administrativos y estudiantiles. Para ello, en primer lugar, necesariamente, tiene que ser participativa, puesto que si las personas son el objeto del cambio que buscamos, también deben ser sujetos gestores de ese cambio. En segundo término, debe ser asumida como un proceso global y extendido en el tiempo, dado 
que no lograremos transformar un todo evaluando y cambiando sólo una de sus partes. De ahí la necesidad de establecer una ruta, trazar un camino crítico a recorrer, efectuar un enfoque sistémico y reconocer que estamos ante un proceso de mediano y largo plazo, aceptando su complejidad y su carácter colectivo.

Tomando en cuenta las características de los modelos aplicados en diversos países de Europa, Norteamérica y Latinoamérica, según los analiza Roberto Ruiz (1998), así como la experiencia adquirida por el IESALC en los casos de la Universidad de la República del Uruguay, la Universidad de Tarija (Bolivia), la Universidad de Costa Rica, la Universidad de los Andes (Venezuela) y la Universidad Central de Venezuela, creemos que en nuestra región los procesos de evaluación sólo deben ponerse en marcha cuando se cumplan determinados requisitos y tienen que incorporar algunas fases o etapas imprescindibles para asegurar su buena marcha.

Los requisitos indispensables serían: la demostración de interés de los dirigentes y su compromiso a largo plazo; la evidencia concreta de una real motivación interna y de una atmósfera de confianza; la adquisición de capacidad técnica para el manejo de métodos de evaluación; la existencia de un sistema de información adecuado y ágil; y la disponibilidad de recursos humanos y financieros suficientes.

Las fases o etapas imprescindibles serían: la sensibilizaciópara que todos los integrantes del demos institucional se aboquen al proceso; la autoevaluación, para generar las visiones que se tienen sobre lo que cada cual hace; la evaluación exten por pas, para contrastarla con la a utoevaluación,hentificar objetivamente las áreas a corregir y definir el plan de transformación; la toma de decisionepara concretar los cambios; y la re-evaluación peranente,para asegurar la continuidad y la buena orientación del proceso. Estas etapas han sido detalladamente reseñadas por Roberto Ruiz (1998), en cuyo trabajo nos basamos para describirlas someramente hoy.

La a utoeva lua ciódhebe comenzar por el diseño y la organización de las actividades que habrán de conducir al diagnóstico de la situación institucional. Ello supone: (i) configurar el grupo organizador del proceso; (ii) seleccionar la estrategia, especificando claramente los objetivos y las metas; (ii) establecer un cronograma de trabajo; (iv) asegurar el pleno funcionamiento del sistema de información; (v) efectuar la estimación de los recursos necesarios.

Esta fase debe continuar con el estudio de la situación institucional en grupos de trabajo, incluyendo en él: (i) la comparación de la misión de la institución con los resultados de su trabajo; (ii) la descripción de la composición y el funcionamiento de los cuerpos estudiantil y profesoral; (iii) el análisis del plan de estudios; (iv) la revisión del proceso de enseñanza, sus métodos y su efectividad; (v) el examen de los servicios estudiantiles; (vi) la descripción de 
las bibliotecas, equipos, laboratorios, plataforma informática y otros servicios de apoyo académico; (vii) el perfil y la productividad de la investigación, sus recursos, áreas de fortaleza y necesidades; (viii) los servicios administrativos y su personal; (ix) los estilos de dirección (toma de decisiones) y formulación de políticas; y (x) la administración de los recursos.

La etapa culminará con la elaboración del informe de autoevaluación, que será redactado por el grupo coordinador, exponiendo, en forma breve y fácil de leer, los resultados de cada aspecto estudiado y las propuestas emanadas del estudio realizado. El documento debe contener recomendaciones concretas dirigidas a superar las debilidades, resolver los problemas y consolidar las fortalezas.

La evaluación externa será realizada por personas cuya trayectoria académica, profesional e intelectual los haga acreedores de una reputación ampliamente reconocida en la materia que van a evaluar ("pares académicos"). Se procurará que sean objetivos e imparciales con respecto a la institución anfitriona, siendo condición insoslayable que acepten el esquema conceptual de evaluación aplicado en la ocasión. Sus nombres deberían surgir de una lista aceptada por la institución bajo revisión, siendo conveniente que procedan de instituciones de nivel similar a la estudiada y que no formen parte de la comunidad académica local.

Los miembros de esta comisión evaluadora deben disponer de las pautas de evaluación, el informe de autoevaluación, el libro descriptivo de la institución, el catálogo de cursos y copias de todas las normas y acuerdos que rigen el funcionamiento de la institución. Antes de la redacción final de su informe, los pares deben realizar una exposición oral de sus conclusiones ante los grupos de dirección que han participado en el proceso de autoevaluación con el fin de matizar o completar el informe final.

La revisión por pares tiene tres objetivos básicos: (i) analizar el informe de autoevaluación, mejorándolo y enriqueciéndolo con nuevas sugerencias; (ii) constituir un grupo de presión para que se pongan en práctica las recomendaciones y se efectúen los cambios; y (iii) dar una opinión independiente que constituya garantía política de su imparcialidad.

La re-evaluaciónsegún (DIAS SOBRINHO, 1998), "es al mismo tiempo una meta-evaluación, pues evalúa el proceso realizado y articula el futuro de ese proceso en un nuevo nivel; es una síntesis que resulta de las dimensiones internas y externas; es un organizador de las metas y medidas que institucionalmente deberán ser adoptadas como consecuencia de la evaluación ... es un elemento fundamental para superar las evaluaciones episódicas e instaurar la cultura de evaluación."

Hecho de esta manera, el proceso resulta formativo permitiendo, realmente, determinar si una institución usa bien sus recursos, si está ofreciendo una educación de calidad y si su función es socialmente relevante. El IESALC 
se ha comprometido a desarrollar modelos autóctonos basados en estos parámetros y nos llenaría de satisfacción que la UNICAMP contribuyera a construirlos formando, con todo el rigor que exige un posgrado académico de alto nivel, los especialistas necesarios para ponerlos a prueba en los países de la región que consideren de ineterés utilizarlos para merjorar la calidad y pertinencia de sus sistemas de educación superior.

¿Qué podemos ofrecer a la UNICAMP en esta aventura? Primero, el caudal de conocimiento institucional acumulado a lo largo de más de veinte años de investigación y trabajo con los problemas de la educación superior en la región. Esto incluye una extensa base de información y de recursos bibliográficos, resultados de experiencias para hacer comparaciones y un plantel de recursos humanos altamente calificados.

Segundo, el acceso a la capacidad profesional que existe en la región. Estamos identificando especialistas que han hecho ya trabajo innovador en el tema de evaluación y los estamos convocando a este esfuerzo conjunto de desarollar modelos alternativos que puedan responder a las necesidades de las instituciones de la región.

Tercero, la posibilidad de estudiar, en forma comparada, los procesos de evaluación que, sobre las bases conceptuales anteriormente mencionadas, el Instituto está impulsando en la Universidad de Tarija, Bolivia; en la Universidad de Costa Rica; en la Universidad de la República del Uruguay; y en la Universidad Central de Venezuela.

Cuarto, y muy importante, la UNESCO ofrece un terreno de neutralidad, objetividad y confianza que hoy no es fácil de ubicar para llevar a cabo un proceso tan sensible como lo es la evaluación institucional. La Organización tiene probada capacidad de interacción y mediación con gobiernos, tanto a nivel de los ministerios como de los órganos parlamentarios, con las instituciones educativas, los organismos internacionales, las ONGs y otras organizaciones de la sociedad civil.

Por todo ello, y a partir de las demandas que sobre nuestra oficina vierten tantas instituciones que quieren poner en marcha su propia transformación, hemos creido oportuno apoyar la iniciativa de la Facultad de Educación de la Universidad de Campinas, en el sentido de crear un programa de posgrado, dedicado a la evaluación de la educación superior. Pensamos que ese programa tendrá un alcance regional, promoverá la cooperación interinstitucional, realizará investigación comparada, y podrá interactuar positivamente con esos verdaderos laboratorios de experimentación que constituirán los proyectos piloto de transformación institucional que el IESALC viene apoyando en varias universidades de la región. Creemos que de este modo se podrán gestar nuevas generaciones que se acerquen a este problema con enfoques enraizados en la defensa del derecho de nuestros pueblos a una educación superior socialmente pertinente y de la mejor calidad. 


\section{Referências}

ATTALI, J. et al. Pour un modèle éuropéen d'enseignement supérieur. Ed. Le Monde, Paris, 1998.

UNESCO. La educación superior en el siglo XXI: Visión y acción. UNESCO, Paris, 1998.

CRESALC. Hacia una nueva educación superior. Colección Respuestas, No. 4. Ed. CRESALC/ UNESCO, Caracas, 1996.

CRESALC. La educación superior en el siglo XXI.Visión de América Latina y el Caribe. Colección Respuestas, No. 5, Ed. CRESALC/UNESCO, Caracas, 1997.

DELORS, J. et al. La educación encierra un tesoro. Informe a la UNESCO de la Comisión Internacional sobre la Educación para el siglo XXI. UNESCO, Paris, 1996.

DIAS SOBRINHO, J. Avaliacão Institucional da UNICAMP. Editora da UNICAMP, Campinas/Imprensa Oficial do Estado S. A., IMESP, São Paulo, Brasil, 1994. luación de la educación superior en Brasil.

DIAS SOBRINHO, J. Evaluación de la Educación Superior en Brasil. Documento de trabajo preparado para la reunión de San Juan, Puerto Rico, 1998.

RUIZ, R. Evaluación académicay educación superior. Versión multigrafiada. Fundayacucho, Caracas, 1998. 